\title{
A Mathematical, Graphical and Visual Approach to Granular Synthesis Composition
}

\author{
Fernando Falci de Souza, Adolfo Maia Jr. \\ Universidade Estadual de Campinas | Brazil
}

\begin{abstract}
Resumo: Neste trabalho apresentamos um método para composição com Síntese Granular baseado em modelagem matemática do gesto musical. Cada gesto é desenhado como uma curva gerada com um particular modelo (ou função) matemático e codificado em um script MATLAB. Os gestos podem ser determinísticos, definidos por funçôes dependentes do tempo, desenhados à mão, ou ainda gerados aleatoriamente. $\mathrm{A}$ informação paramétrica dos gestos é interpretada através de mensagens OSC por um sintetizador granular (Granular Streamer). A composição musical é então realizada com os modelos (scripts) escritos em MATLAB e exportados para uma partitura gráfica (Granular Score). O método é adequado para permitir análise estatística do fluxo sonoro granular e composição musical final. Ainda sugerimos um meio de criar fluxos sonoros granulares baseados em parâmetros granulares correlacionados.
\end{abstract}

Palavras-chave: Síntese Granular, Gestos Sonoros, Partitura Gráfica, Modelagem Matemática.

\begin{abstract}
We show a method for Granular Synthesis Composition based on a mathematical modeling for the musical gesture. Each gesture is drawn as a curve generated from a particular mathematical model (or function) and coded as a MATLAB script. The gestures can be deterministic through defining mathematical time functions, hand free drawn, or even randomly generated. This parametric information of gestures is interpreted through OSC messages by a granular synthesizer (Granular Streamer). The musical composition is then realized with the models (scripts) written in MATLAB and exported to a graphical score (Granular Score). The method is amenable to allow statistical analysis of the granular sound streams and the final music composition. We also offer a way to create granular streams based on correlated pair of grains parameters.
\end{abstract}

Keywords: Granular Synthesis, Sound Gestures, Graphical Score, Mathematical Modeling 
Through all the twenty century many composers and scientists were concerned about the exploration of new sounds, extending the sound possibilities of musical instruments $\mathcal{L}$ expression through the so called extended techniques, or creating new ones, or even artificially producing them through electronic devices. An early example of this trend is Varèse's Ionisation (1929), virtually the first work for percussion ensemble only, in which he explores the expressive power of pitched and unpitched percussion instruments and even used an unexpected siren (FRANÇOIS, 1991). The Fifties were rich in newness for music expression. Pierre Schaffer, together with Pierre Henry, created the Group de Recherche de Musique Concrète (GRMC) where they lead important experiments on generation and organization of sounds including music composition. Concurrently, in Cologne (Germany), Werner Meyer-Eppler and collaborators founded the Studio for Electronic Music at Westdeutscher Rundfunk (WDR) where they also lead research and composed pioneering musical works in Electronic Music. On the other hand, computers were used for the first time to compose music by Lejaren Hiller in his Illiac Suite (1957) (HILLER and ISAACSON, 1959). Later Max Mathews pioneered the use of computers for sound synthesis at Bell Telephone Labs, an important breakthrough in music technology. A good historical approach of sound synthesis can be found in (MANNING, 2011).

In 1949 Dennis Gabor presented his idea on the creation of a "quantum of sound", later best known as "granular sounds". In the musical milieu the composer Iannis Xenakis was the first one to use the idea of "grains of sounds" in his work Analogique B (1959). It can be played with its instrumental counterpart Analogique $A$ to form a mixed piece named Analogique $A$ et $B$ (DI SCIPIO, 2005; XENAKIS, 1992). Later, the composers Curtis Roads and Barry Truax made extensive studies in techniques for Granular Synthesis developing several sound synthesis systems as well as composing several musical works. The state of the art by 2001 is presented in Roads' book Microsound (ROADS, 2001).

In 1988 Barry Truax developed a system for real time granular synthesis (TRUAX, 1988). Since then, more complex and encompassing systems had been developed and the trend continued in 21st Century with the development of several integrated sound systems for multimedia. Performances now are very diverse since multimedia can be attached to many different presentations such as, live pop music shows, symphonic orchestra and other ensembles, multiple internet 
concurrent presentations, and so on. So, we present our granular synthesis-based system which can rise new ideas for multimedia performances. In Section 3.4 we present a short review of some comparable systems for granular synthesis.

The structure of this work is as follows. In section 2 we briefly described the basics of Granular Synthesis with some historical context. In Section 3 we describe our software model for Granular Synthesis. In Sections 4 we describe our experiments in Granular Synthesis Composition via mathematical models written in MATLAB scripts. In Section 5 we suggest some other mathematical models which can be coded in MATLAB. In Section 6 we show some artistic works we have presented using our models and granular system device. Finally, in Section 7 we make some conclusions and perspectives for future work.

\section{A Short Introduction to Granular Synthesis}

Granular Synthesis was discovered by the Nobel Prize winner physicist Dennis Gabor inspired by Heisenberg's Uncertainty Principle of the Quantum Mechanics. Gabor showed that, formally, any sound can be represented as a set of acoustic cells with simple spectral content (sine waves) and duration of about 10 to $200 \mathrm{~ms}$ (GABOR, 1947). Strictly and roughly speaking granular synthesis is nothing more than a mathematical decomposition of a waveform into a vector basis of a Hilbert Space just as Fourier decomposition. This means that, whereas a waveform $W(t)$ can be written in a Fourier basis as a trigonometric series

$$
W(t)=\sum_{n=0}^{\infty} a_{n} \cos \omega_{n} t+b_{n} \sin \omega_{n} t
$$

where $\omega_{n}$ are the spectral frequencies (or Fourier partials), $a_{n}$ and $b_{n}$ are amplitudes and $B=$ $\left\{\cos \omega_{n} t, \sin \omega_{n} t\right\}$, for $n=0,1,2, \ldots$ is a trigonometric basis for the Hilbert Space of all possible waveforms, a Gabor's decomposition reads

$$
W(t)=\sum_{n=0}^{\infty} A_{n} g_{n}(t)
$$


where $A_{n}$ are amplitudes and each $g_{n}(t)$ is a so-called Gabor's Wavelet which has the form

$$
g(t)=e^{-a^{2}\left(t-t_{0}\right)^{2}} e^{i \omega_{0}\left(t-t_{0}\right)}
$$

This represents, an elementary "granular" waveform centered at time-frequency point $\left(t_{0}, \omega_{0}\right)$ satisfying the Heisenberg's Inequality

\section{$\Delta \omega . \Delta t \geq 1$}

where $\Delta t$ and $\Delta \omega$ are, respectively, the uncertainty to measure the duration and frequency with any device, including, digital filters or even, of course, the human ear.

FIGURE 1 - A typical wavelet centered at point $\left(t_{0}, \omega_{0}\right)=(0,0)$

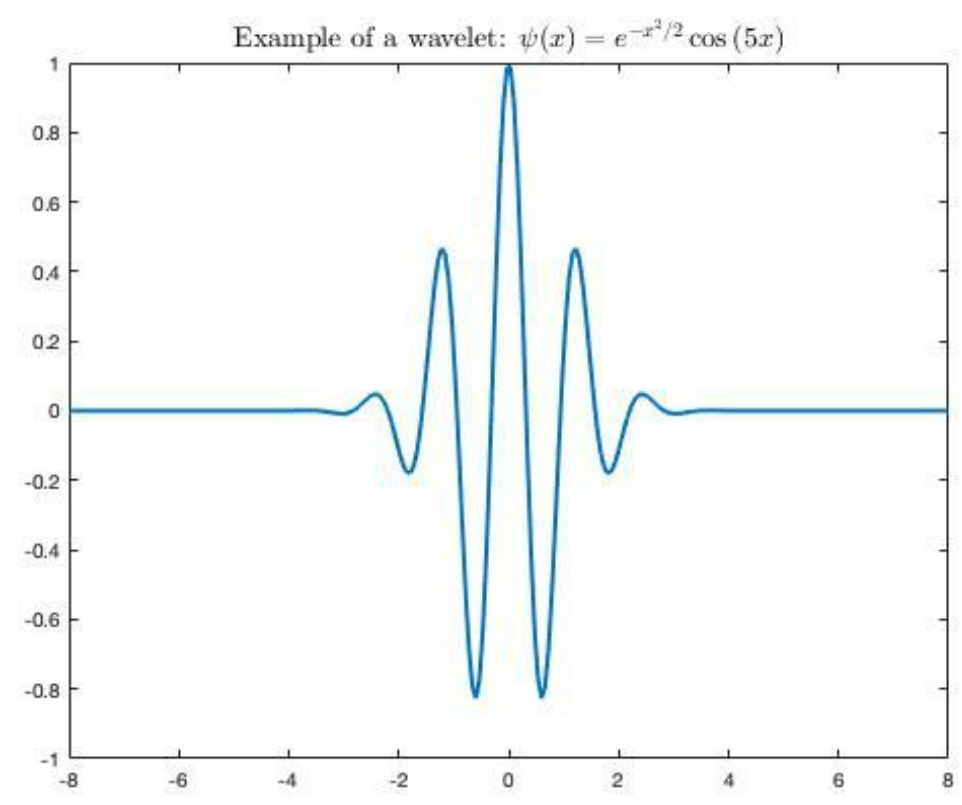

Source: authors

The Gabor's wavelets have minimal product of uncertainties, namely, $\Delta \omega . \Delta t=1$ and are also named acoustical quanta. A representation of an elementary sound grain is showed in Figure 1. It shows a typical Gabor's wavelet centered at $\left(t_{0}, \omega_{0}\right)=(0,0)$. An important property of the set of Gabor wavelets, as many other different basis of representation of digital signals is its behavior on 
large times: they exponentially decrease asymptotically to zero or even are equal to zero outside a finite interval. In the example of Figure 1 this is achieved multiplying the signal $\cos (5 x)$ by the decreasing exponential function $e^{-x^{2}}$. This also implies the wavelet has finite energy. It is the size of the spectral envelope that allows the sound granulation theoretically with arbitrarily small durations. In addition, the Fourier Transform of a Gabor's wavelet in time domain is a Gabor's wavelet in frequency domain. So, Gabor wavelets can be viewed both in time or frequency space.

FIGURE 2 - The Time x Frequency Gabor's Decomposition. The minimal area of the rectangle is equal to 1.

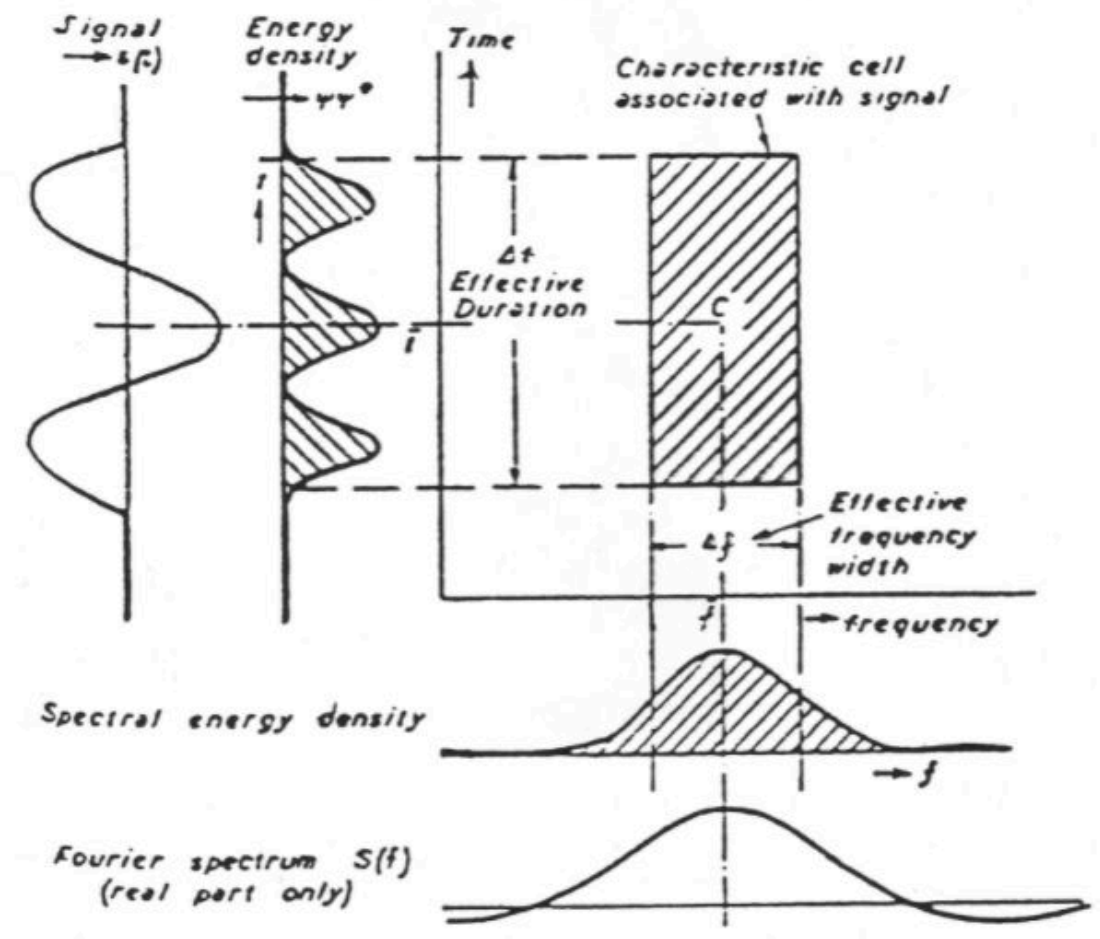

Source: Gabor, D. 1947 paper. See GABOR (1947, p. 591)

Figure 2 is the original one from Gabor's paper (GABOR, 1947). We can see that the signal was "decomposed", or viewed, in time domain, with duration $\Delta t$ and, in frequency domain, as a bell shape envelope with a frequency bandwidth $\Delta \omega$ which ensures the signal has finite energy. The area of the decomposition $\Delta \omega . \Delta t$ is a dimensionless quantity, with minimal value equal to 1 , which is the Gabor's grain of sound. Heisenberg Inequality for Gabor wavelets or, more generally, for any grain whose duration is a finite interval can be written as 


$$
\Delta \omega=\frac{1}{\Delta t}
$$

This equation can be interpreted in the following way: if you have a grain with very short duration you get a spread frequency interval, so you can't measure its spectral content with a good precision and vice-versa, to get precisely the frequency content of a grain it must have a large duration. This is also an important property of Fourier Transforms.

Figure 3 shows different time-frequency cells (rectangles) where the Gabor wavelets are defined. So, we can have cells with different widths for time and frequency but with the same area. All of them are representation of grains of sound with their own durations and spectral content.

FIGURE 3 - Diagram showing the Gabor's cells (grains) in the space Frequency $x$ Time. Each rectangle is centered at a particular frequency $\omega_{0}$ and time $t_{0}$ and its area of satisfies the Heisenberg's relation $\Delta \omega . \Delta t \geq 1$.

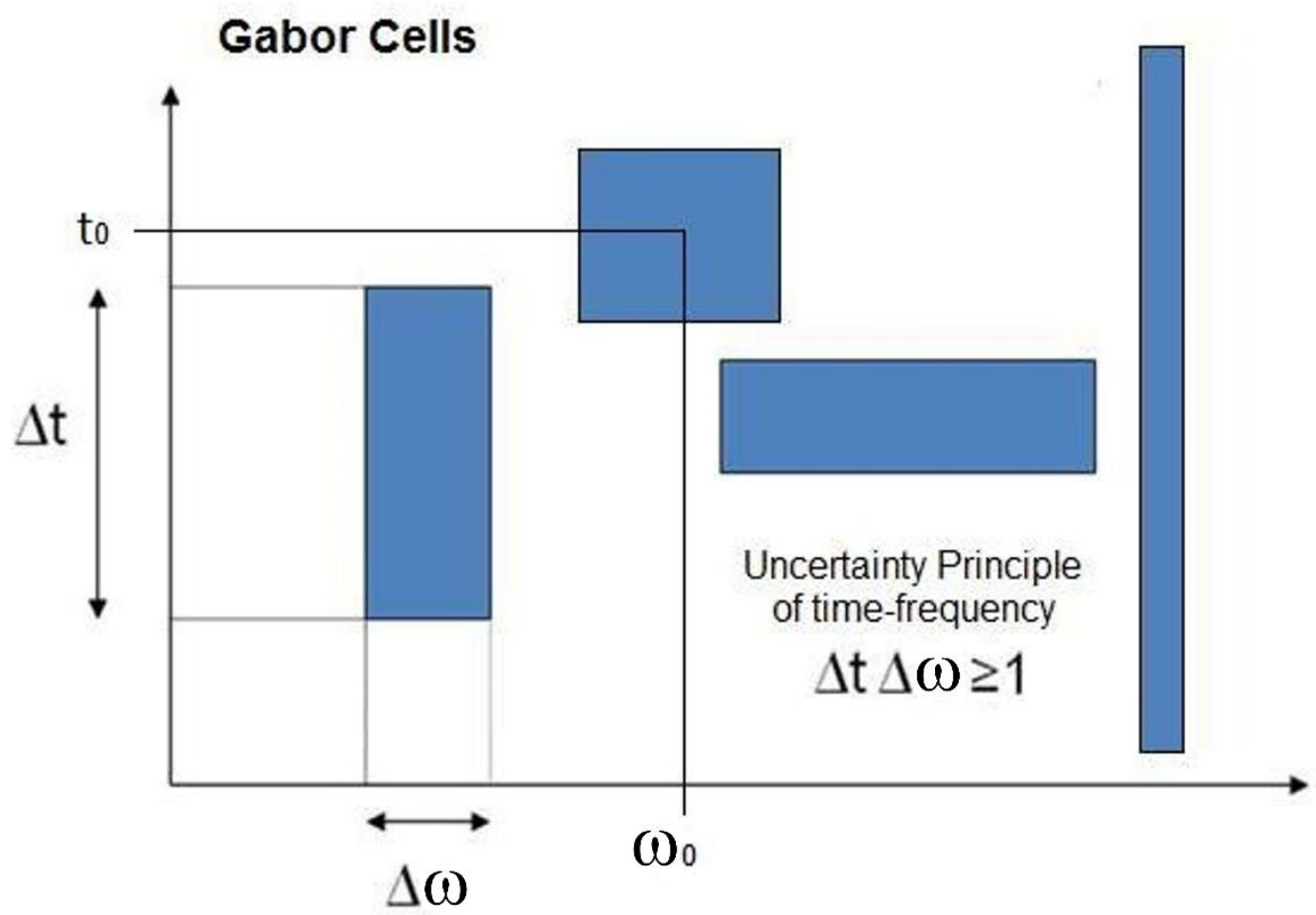

Source: authors

Gabor's wavelets are used in many areas such as Sound Analysis, Image Processing, Communications among many other. For a comprehensive approach Gabor's Theory with applications on Granular Synthesis see (ROADS, 2001, Chapter 3). 
However, as mentioned above, Gabor wavelets are only an example of an elementary grain of sound. In fact, there exist an infinite number of possibilities to create grains of sound. For example, if we don't consider the Heisenberg inequality we can construct granular streams with sounds with very short duration, say of order of milliseconds. For many practical applications this is perfectly fine and, in fact, many musicians use a technique named granulation that does exactly this, take a digital sampled sound and split it in many others with very small durations. A time (or frequency) envelope must be provided to all grains. In fact, this very "informal" way to define sound grains is just the Roads one when he considers Granular Synthesis "A grain of sound is a brief microacoustic event, with a duration near the threshold of human auditory perception, typically between one thousandth of a second and one tenth of a second (from 1 to $100 \mathrm{~ms}$ ). Each grain contains a waveform shaped by an amplitude envelope.” (ROADS, 2001, p. 86)

The drawback of this kind of technique is, in general, lack of fine internal control of the spectral content of the grains. A mathematical approach allows this control, however the spectral wealth can be compromised since it's very difficult to sculpt a wished sound wave as, for example those ones of musical instruments. Between these two extreme approaches there are plenty of room for many possible hybrids methods to generate, as Roads named them, microsounds.

Most composers of electroacoustic and electronic music make use of random generation and control of sets of grains. Words like clusters, clouds are now commonly used denoting the easy composer's acceptance of relying the micro-control of granular sound structures on probability distributions, due to the practically impossible human task to define each sound grain in a particular way in a flux of thousands of them. Roads resumes the problem from his own experience "The first task I confronted was automating the grain generator. Integral to Xenakis's conception of granular synthesis was the notion of global stochastic control.” (Roads, 2001, p. 303).

We present here a mathematical approach to generate sound streams with a large number of grains based on parameters as frequency and duration which in our model are time dependent. In our model, sound structures can be thought as sound gestures defined by time functions of the grain's parameters. This approach allows to have a deterministic approach of sound gestures, that is, the same sound stream can be exactly repeated irrespective of the quantity of grains. Nevertheless, we 
also present some probabilistic models like, for example, that one with frequency and duration parameters satisfying a given mathematical relation.

From the point of computational/algorithmic point of view our models are written as MATLAB scripts whose output are sets of strings with 10 parameters which can be read by the composition software Granular Score and interpreted as an OSC message by Granular Streamer. In other words, MATLAB scripts, or codes, are "scores" of sound gestures and the two AVGS (Audio Visual Granular Synthesis) software are interfaces: Granular Score is the graphical score, and Granular Streamer is sound interface. The details of our software we present briefly below.

\section{The Engine: Generating Granular Streams from Structured Text Files}

Our software AVGS (Audio Visual Granular Synthesis) is a Java based software for Audio and Image Composition. Its audio part is controlled by a program we named Granular Streamer which is in charge for the creation and control of granular sound streams. Its graphical counterparts are:

a) an interface named Granular Score, which works like a graphical score allowing a higher level and easy control of the Granular Streamer.

b) an interface for a visual animation of sound streams named Granular Drawer.

\subsection{Granular Streamer}

Granular Streamer is the main program of the software set above mentioned. It has the instructions for configuring sound devices as well global variables. It is capable of synthesizing a large number of simultaneous streams and, for each stream, a different type of wave, envelope and maximal number of grains per second must be specified. Figure 4 shows its interface of control. The button Play turns it activate for a session with Granular Score and Granular Drawer. 
FIGURE 4 - An example of Granular Streamer Configuration Window

\begin{tabular}{|l|l|l|}
\hline \multicolumn{1}{|c|}{ Default } & & \\
\hline
\end{tabular}

Source: authors

Notice that Granular Streamer interface has buttons to load and save configurations and to play and stop the sound synthesis, but it doesn't have any built-in controllers to drive the parameters in which each stream is going to generate sound grains. Actually, those controls are implemented in secondary modules in many ways, to mention a few, the composer can:

- create a patch in Pure Data or MAX/MSP with sliders or any other kind of interface and send Open Sound Control (OSC) messages in real-time to Granular Streamer;

- use Granular Score to position manually each grain in the desired time with specific chosen parameters;

- create a structured text file (formats file.txt, or its own file.gsf) with a particular format.

In our approach a structured text file is formed by rows with 10 entries of numerical data (parameters) sided by a left text entry with the word "grain" and by a right one with the semicolon symbol “;” as shown below: 
Each row, as the above one, has information data to define one sound grain. For each grain, the data, from left to right, are values of the following parameters, choose by the user:

grain: a fixed word at the beginning of the row indicating the data on the right are associate to a grain;

time: this is the time of the attack of the grain from the beginning of the synthesis or start of the piece of music;

stream: this is an integer value $(0,1,2$, or 3$)$ which indicates the stream number. Granular Streamer can run many simultaneous streams, which is configured to synthesize grains with a specific type of wave and envelope;

frequency: the frequency (in Hertz) of the wave inside the grain;

duration: duration of the sound grain in milliseconds;

amplitude: amplitude of the wave inside the grain with a value from 0 to 1 ;

position $\mathbf{X}$ : position of the grain in stereophonic (left to right) with values from -1 to $1 ;^{1}$

position Y: this parameter does not affect sound synthesis, but when Granular Streamer is linked to Granular Drawer, this parameter is the vertical position in which the grain will be plotted on the screen; ${ }^{1}$

density: this parameter does not affect sound synthesis when it is controlled from a text file. It indicates the density of grains (number of grains generated per second) and it will be by-passed by Granular Streamer; ${ }^{1}$

offset: if the grain stream was specified to use a wavetable instead of a fixed wave type like sine, square, etc., this parameter indicates the position to start reading the waveform in a table with values between 0 (beginning of the table) and 1 (end of the table), see details below in the topic Granulation of Sound; ${ }^{1}$

offadd: this parameter does not affect sound synthesis when it is controlled from a text file, but it specifies an automatic shifting of the offset. In this case it will be by-passed by Granular Streamer; ${ }^{1}$

\footnotetext{
${ }^{1} 0.0$ for our actual experiments.
} 
Any text rows structured as the above format can be read by the AVGS, that is it accepted as a dataset. In our approach this is provided by a mathematical software of general application, namely, MATLAB. Of course, other mathematical software can be used for this purpose. However, for composition with a great number of grains it is better to have an automatic grain generator, that is, a set of few parameters which are used to generate and control particular generation of sets of grains with specific characteristics such as frequency, duration, spatialization, and so on. In addition, if two or more parameters have some functional interdependence, the generated sets of grains can evince a particular sound structure which can be useful for algorithm composition. In our model we used MATLAB in order to do this task.

Granulation of Sound is a general term for methods designed to get granular objects (grains) used in composition. Grains are obtained through the so-called Granulation Process (ROADS, 2001, p. 98) and gets its name because, unlike the synthesis, in which the sound grains are synthesized using mathematical functions, in the Granulation Process an audio file is broken into pieces of very short durations which are enveloped by an amplitude envelope as, for example, a Gaussian. In short, the waveform of the grain is obtained directly from the audio recording.

Besides the usual parameters that specify values for individual grains or for a stream of grains, a new parameter is added to indicate the point inside the audio file in which the grain waveform is extracted, that is, the "reading point" or offset. This new parameter, in turn, can be moved dynamically through a second parameter indicating an "increment for the reading point", which we named as offadd. This increment specifies a displacement of the reading point forward or backward in the sound file. The direction and speed of this movement results in an effect of stretching, shrinking or even "reversing" the time of the recorded sound without changing its frequency. It is also important to stress that in the specific case of granulation, the parameter frequency does not indicate an absolute frequency of the grain, but instead, it is now used as an indicator of "speed of reading" of the sound file samples, resulting in a changing of the sound pitch to lower or higher frequencies without modifying its duration. 


\subsection{Granular Score}

In general, a graphical score is a visualization of sound information with a specific mapping that favors the analysis, creation and editing of a composition. In our research, we found motivation in some well-known graphical scores, such as the classic aural score of the Gyorgy Ligeti's electroacoustic composition Artikulation created by Rainer Wehinger (WEHINGER, 1970). In this work, Wehinger uses different symbols for each type of sound articulation present in Ligeti's music and then arranges these symbols on a timeline left to right, as in a traditional score. Wehinger is very clever in his choice of graphic symbols making a kind of "graphology psychoacoustics", that is, the plot is close to what common sense accepted for each type of sound.

Also, in the work of the Greek composer Xenakis, the UPIC system implements a graphical score for electroacoustic music composition from user-made designs. In 1978, Xenakis used this system to compose the Mycenae-Alpha work (SQUIBBS, 1996). Currently, there is a wide variety of systems that implement different graphical scores as, for example, Iannix, Hyperscore, HighC, Music Sketcher, among others (THIEBAUT, 2001).

In order to have a flexible and easy control of granular structures for composition, we implemented the system Granular Score (see Figure 5), a graphical score that allows the organization and arrangement of granular objects in deferred time with great precision of the control of individual or groups of grains.

FIGURE 5 - Granular Score Interface showing the control panel and some selected granular sound gestures.

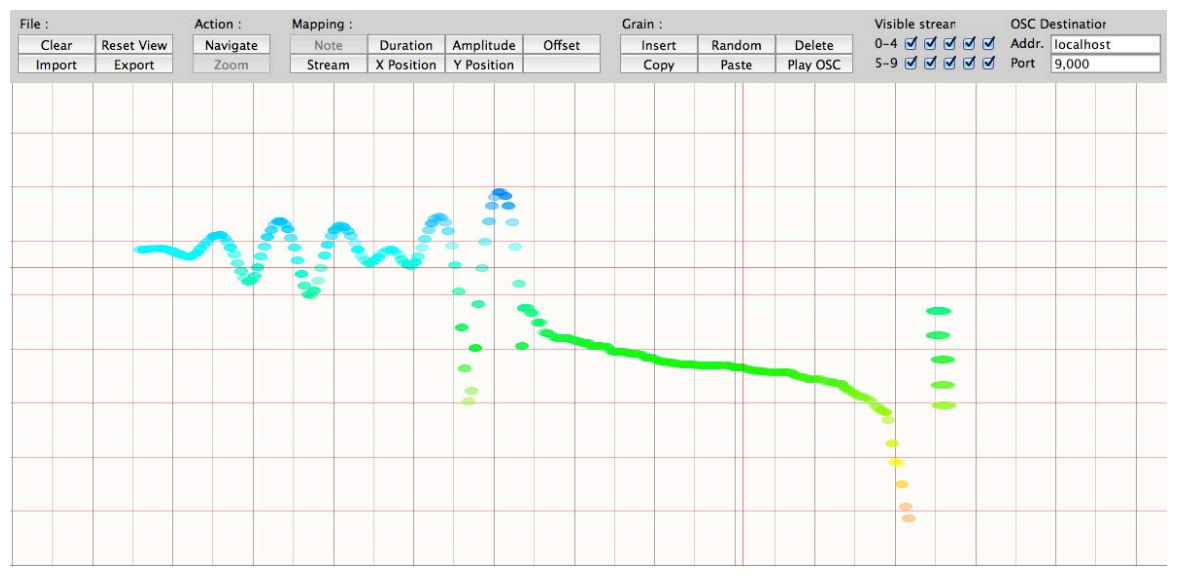

Source: authors 
The upper part of the interface consists of control buttons and at the bottom, that occupies most of the window, is located the graphical score. To allow a clear view of sound information, grains of sound are mapped one to one in visual grains. The frequency of a grain waveform is mapped to the color, amplitude to transparency, and duration to its width of the visual grain. The visual grains are placed in the score from left to right (horizontal axis) as its time position in the arrangement like a conventional score. The vertical axis can be configured by the user to represent any value of the grain, by clicking the corresponding button in the "Mapping" section of the interface, to select “Note” (frequency), “Duration”, “Amplitude”, “Position X”, "Position Y”, “Offset” and "Stream Number”. Thus, different spaces can be viewed one by one, in the same window. The user interacts with the system largely through the mouse, in two distinct modes, navigation and zoom. In navigation mode, the mouse is used to scroll around the score and, in zoom mode, to control the level of detail seen at the score. Although simple, the zoom tool is very powerful because it allows a complex granular structure to be viewed both macroscopically, when the display area of Granular Score is occupied for several seconds, and microscopically when the area is occupied by grains with few milliseconds of duration, thus enhancing the analysis and manipulation of materials at different time scales.

With this system, the user can create new scores from the scratch or import a grains file created previously or produced by any software following the specified syntax. In our first experiments, we used MATLAB random functions, according to a stochastic model, to generate our structured text files and grains streams. MATLAB is suitable to quickly compute matrix operations allowing a deterministic approach for grain streams generation. In addition, a score can also be performed in real time by clicking the "Play OSC" button. The Granular Score will send Open Sound Control (OSC) messages through the network to the Granular Streamer, which will play grains of sound. In order to implement functions for sending and receiving OSC messages we used a package of libraries in JAVA developed by Chandrasekhar Ramakrishnan (RAMAKRISHNAN, 2021). With the mouse the user can select and edit the position of a grain or a group of grains. A horizontal displacement changes the time of the grains in the arrangement, and a vertical displacement changes the parameter value of the grain that is selected on the $y$ axis. The interface also has functions to delete, copy, paste, and insert new grains. In our experiments we took a combination of automation 
by a deterministic model to generate grains and the freedom of control them along time, in a mixture of reasoning and intuition according to the principles of formalized music as settled by the composer Iannis Xenakis (XENAKIS, 1992, p. 22).

\subsection{Granular Drawer}

Complementary to the Granular Streamer, which receives the granular information from the Granular Score and process it as a grain of sound, this system is used to create a visual animation in real time based on grains of image. Most of its functions comes from Processing ${ }^{2}$, a simplified programming language that provides a flexible environment for learning and coding within the visual arts context. Granular Drawer also uses OSC messages to communicate to other systems. In other words, this system implements the Visual Granular Synthesis that, together with the Granular Streamer, results in the Audiovisual Granular Synthesis. Previously in our research we investigated some possible correlations between light and sound (COLLOPY, 2009). On the other hand, cognitive functions of the brain are responsible for the amalgamation of auditory and visual stimulus in multisensorial objects (KAPUCINSKI, 1998). With so many possible forms of audiovisual correspondences, the practice in the visual arts shows that the relationship between sound and image depends fundamentally on an aesthetic decision (CIUFO, 2001, p.2) which in turn is dependent on the possibilities of the system used and the artist's creativity.

Due to the immense possibilities of types of grains and visual ways to find these grains in granular objects larger and more complex, we decided it would not be appropriate or even impossible to create a complete system. Thus, in the current version of Granular Drawer, the user must select an instance of Visual Granular Synthesis from twelve possibilities, each with types of visual grains and specific effects. In one of the available options, the visual grains are represented by semi-transparent colored circles as show in Figure 6.

\footnotetext{
${ }^{2}$ Retrieved from < https://processing.org/> on July 1st, 2021.
} 
FIGURE 6 - Granular Drawer Screenshot with circles representing sound grains. In this version the animated granular flux comes from the bottom.

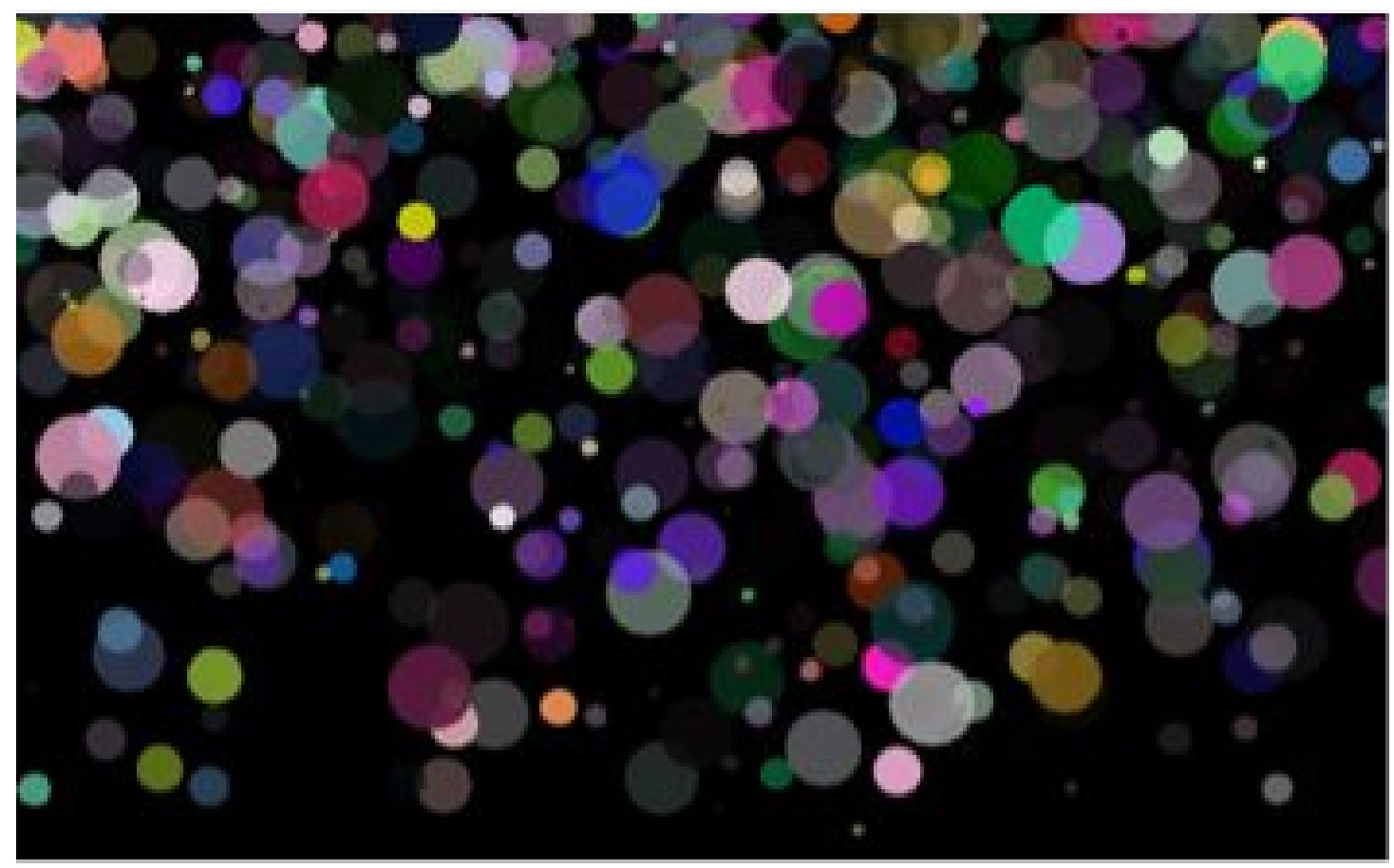

Source: authors

Figure 7 shows a more recent work, named Sao Paulo Granulado (2019), that uses Granular Drawer with concrete images reproducing an artistic view of the landscape of the city.

FIGURE 7 - Granular Drawer Screenshot with visual grains with concrete images associated with sampled grains of sound.

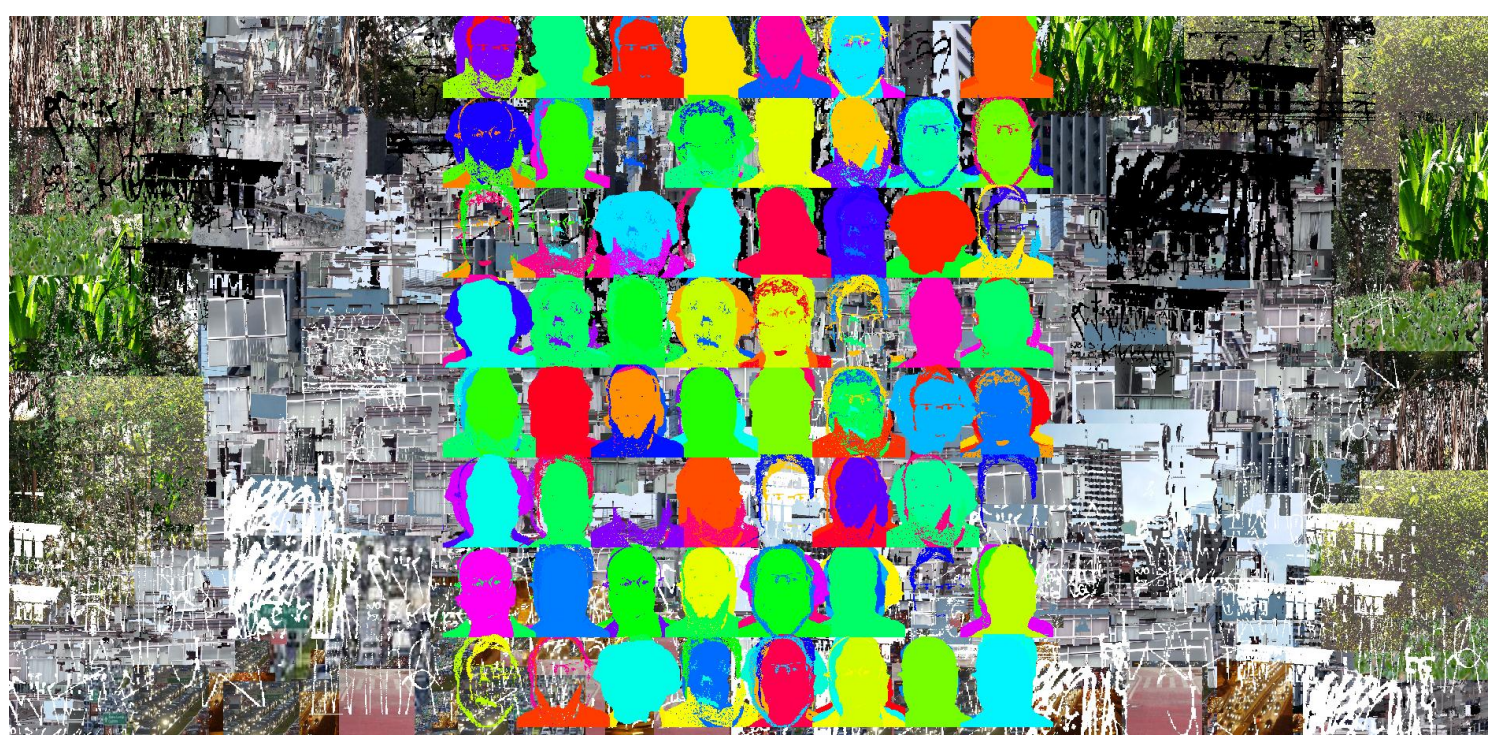

Source: authors 


\subsection{Other Comparable systems}

Since we began our research in Granular Synthesis during our master project in 2008, we tested and analyzed many systems. To name a few: Granulator by Nicolas Fournel (currently offline), Granulab $^{3}$, Kenaxis ${ }^{4}$, Chaosynth (MIRANDA, 1995). We have also experimented with music programming environments like CSound and JMusic, as well as implemented patches in Pure Data and Max/MSP. Many other options, stand-alone or not, are available for those interested in Granular Synthesis. ${ }^{5}$

Many of those systems perform similar functions. Some return simple and specific outputs specific, other are more complex with greater variety of audio outputs. Our system can work with sound grain clouds without any restriction of calculation since it is off time. In Granular Streamer, for instance, we have programmed the synthesis at the sample level, calculating each grain's parameter with our algorithmic implementation and gradually we increased the functionality of the system. Some advantages of doing this, is to avoid some overhead functions that might be part of other software. So, our system is independent of any third-party software. We think this is important in case we need to customize the software, and last but not the least, we have stand-alone tools when using our systems out of the academic context considering commercial applications.

\section{Experiments in Free Composition}

\subsection{Generating Compositional Material: Structured Text Files via MATLAB Scripts}

The main script is named mat2stream. Since we have ten parameters of control at our disposal, for a stream with $\mathrm{N}$ grains, its output is a $\mathrm{N} \times 10$ numerical matrix of parameters of control, shortly Control Matrix, which represents a time ordered set, or sequence, of grains. Each row represents a grain. In our examples some of these parameters have 0 value as, for example, spatialization, and so that parameter is not active for the stream.

\footnotetext{
${ }^{3}$ Retrieved from < https://www.abc.se/ re/GranuLab/Granny.html > on July 1st, 2021.

${ }^{4}$ Retrieved from <http://www.kenaxis.com/> on July 1st, 2021.

${ }^{5}$ An extensive list can be found at https://www.granularsynthesis.com/software.php
} 
Now, in order to create a sound structure, the grains of the stream, or equivalently, the lines of the Control Matrix, must be related in some way. So, it is necessary to establish a functional dependence, or relation, between the grains parameters. Of course, there are infinite ways to do that. Nevertheless, for the human ear many different streams are perceived as very similar and thus, for musical purposes, equivalent. This, of course, implies a kind of taxonomy for streams. We propose here a simple and intuitive classification of the streams and present the correspondent algorithm to produce them. In our approach to composition experiments we classify the sound streams in six simple groups: block, cloud, constant jet, increasing jet, decreasing jet, periodic.

\subsection{Some Models for Free Composition}

Composition in our context is a kind of game between a MATLAB script and the Granular Score and the arena is that one settled by Granular Streamer which fix global variables, say, the rules of the game. On the one hand MATLAB scripts produces structured text files, or Control Matrices, generated by mathematical models envisaged by the composer. These files are then imported to Granular Score which in turn generates correspondent granular structures viewed in its graphical interface. If the imported material is useful and matches the aesthetic goals of the composer, he/she can save the session and, on the contrary, the imported material can be deleted, and the composer returns to the MATLAB models. In fact, an arbitrary number of granular structures can be produced and used as fundamental units or, as we named them, basic modular sound musical gestures, which the composer can manipulate at will in each of parameter spaces such as note, duration, amplitude among others. Below we present some examples of mathematical models we have used in our MATLAB scripts. For all models we defined as global parameters: a time interval $[0, \mathrm{~T}]$ and the number of grains $\mathrm{N}$.

a) Sinewave: it is based in a modulated sine model given by system:

$$
\begin{gathered}
f(t)=f_{0}+g(t) \sin (\omega t) \\
g(t)=A t \sin (\omega 0 t)
\end{gathered}
$$


where $g(t)$ is a low frequency modulating function with $\omega 0<<\omega, A$ is a constant and $f_{0}$ is a mean frequency around which the sine function oscillates. Most of granular sound gestures in the Figure 5 were obtained with this simple model. Observe that the composer has, for this particular model, three free parameters $\omega, \omega 0$ and $A$.

b)Linear Glissandi: this is the simplest model with just a linear time function

$$
f(t)=f_{0}+a t
$$

where $f_{0}$ is an initial constant frequency and $a$ is a constant. If $a>0$ we get glissandi with increasing frequency along time, if $a<0$ the frequency is decreasing. Of course, $f_{0}$ and $a$ must be choose such that $f(t)>0$, since there is no negative frequency.

\section{c) Random Frequency Models}

For these models it is necessary to define Probability Distribution Functions in order to generate random number generators with can be used as input of several grain parameters. We have used, for our examples, the following distributions:

$$
\begin{gathered}
p(x)=1 \quad \text { Homogeneous Distribution } \\
p(x)=e^{-a\left(x-x_{0}\right)^{2}}, \text { with } a>0 \text {, Normal Distribution }
\end{gathered}
$$

where the variable $x$ can be any of the grain parameters defined above. The constant $a=\frac{1}{2 \sigma^{2}}$, where $\sigma$ is the Standard Deviation. We can generate, for example, random grain frequencies around a mean frequency of $x_{0}=600 \mathrm{~Hz}$ with standard deviation of $40 \mathrm{~Hz}$. Once we have defined the probability distribution we can use a random number generator, for example with MATLAB, to produce an arbitrary quantity of values of frequencies, one for each grain. Of course, many other distributions can be used, including a defined by the user through a Cumulative Distribution Function. 


\section{d) Frequency $\times$ Duration Linear Model}

As default, frequency and duration are independent variables. Nevertheless, we can relate grains duration $d$ and its frequency $f$ in many ways. A very simple one is just defining a suitable function $d=F(f)$. For example, a linear model as

$$
d(t)=d_{0}+a \cdot f(t)
$$

where $d 0$ is an initial duration, $a$ is a constat, and $f(t)$ is the frequency function. The net effect is a kind stretching $(a>0)$ or shortening $(a<0)$ of grain durations in the stream along time. Observe that the generation of frequencies can be both deterministic or probabilistic.

In general, composition is just a time ordered set of modular sound gestures arranged in a particular way by the composer. When a composition session is finished, the work can be saved, using the export button in the interface, as a text file with extension $g s f$, native of Granular Score or as the usual txt. In general, the electroacoustic music composed with the computer programs described above is a textural one. This means that it is structured as layers of sets of modular gestures and it's not, in general, related to tonal music. The Figure 8 shows part of an experimental composition of ours. Observe that different patterns are clearly recognized showing the combination of diverse modular gestures. Of course, the composer is also free to superimpose several modular gestures and in this way, it is not possible anymore to distinguish clearly the constituent modules.

FIGURE 8 - Extract of an experiment in composition in space time $\times$ frequency showing several structures produced by a Sinewave model.

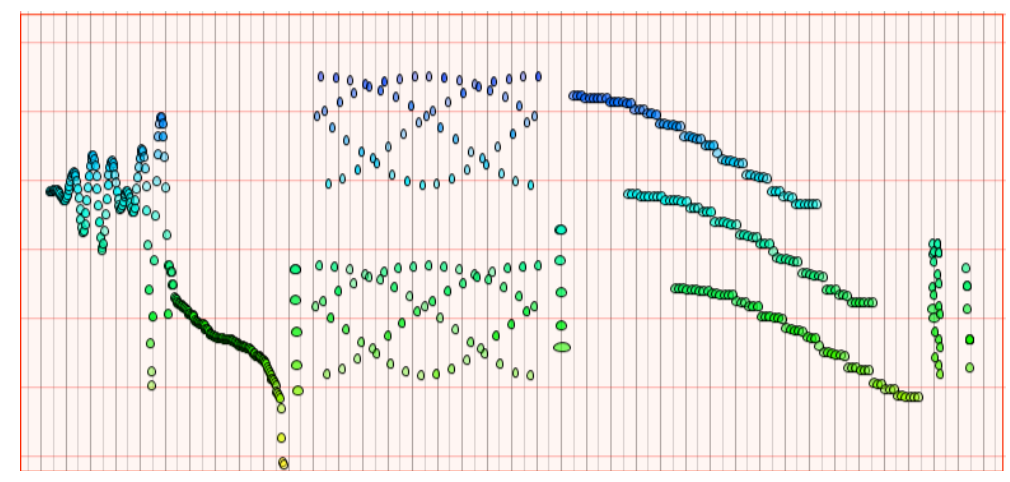

Source: authors 
Working with the three computer programs described above and sets of free parameters the composer can have to her/his disposal amazing surprises of patterns of modular gestures.

\section{Examples of Mathematical Models for Granular Composition}

All saved streams can be considered as sound modular gestures which the composer has to his/her disposal for sequencing, superposition, control of duration, amplitude and other parameters supplied by Granular Score. We think this is a mixed approach resembling, in certain sense, that one by Xenakis (XENAKIS, 1992, p. 22). Granular Synthesis has proper, peculiar, sound characteristics and, clearly, each composer has his/her own style and aesthetics to generate sound structures, to order them in time and compose. Below we show a list of some mathematical models which can be coded as input into Granular Score.

a) Denoting $S$ the multidimensional space of $n$ grain parameters $x 1, x_{2}, \ldots, x_{n}$. The time evolution of a stream can be defined as a curve in $S$ with a set of parametric equations:

$$
\begin{gathered}
x_{1}=x_{1}(t) \\
x_{2}=x_{2}(t) \\
x_{3}=x_{3}(t) \\
: \quad: \\
x_{n}=x_{n}(t)
\end{gathered}
$$

For composition is very important to study which classes of functions can be effectively used. For example, we can test the rich space of periodic functions such as the oscillating Bessel Functions of First Kind.

b) Dynamical Systems like the above one can present chaotic behavior. This is most interesting since, although deterministic, chaotic systems can be macroscopically close to truly random systems. The consequence is granular streams sounding as random. In addition, dynamical systems can also 
present quasi-periodic orbits due to singularities and this behavior can also be mapped in Granular Score furnishing also quasi-periodic streams.

c) Recurrence is also a deterministic approach for defining modular gestures using a recurrence equation such as

$$
z_{k}=f\left(z_{k-1}\right), \quad k=0,1,2, \ldots
$$

where $f$ is a suitable real function. In this model the information, that is, the values of a grain's parameter depend on the values of parameters of the previous grain. In fact, this can be generalized to a dependence of an arbitrary number of previous grains.

d) Another extension, technically more complex, is to use a tablet to draw curves in a window, which represent the parameters of Granular Streamer. Each point of the curve gives rise to a row vector which can be sent to a computer by Bluetooth and saved as grain format to be read by Granular Streamer. In this way musicians can experiment more easily with composition strategies. Alternatively, it's also interesting to create an applet to run on the tablet itself.

e) In many experiments Random Methods produce very similar granular streams, even for large variations of control parameters, as we mentioned above. A further study on the responsiveness of variability of grain streamers under the control parameters of the random model is needed in order to have a useful device for granular music composition.

f) It worth also to mention that we can use mathematical programs like MATLAB in order to get statistical information from a stream or group of streams. This is also interesting for composition since the statistical quantities can be useful, for example, as suitable restrictions imposed by the composer. Statistical analysis can, of course, be performed for any of the models above mentioned. 


\section{Artistic Practice with Granular Sounds}

Xenakis was one of the first composers to work with granular synthesis. Due to the limitations of the analogic technology, he had to do a great effort to slice and reassemble pieces of magnetic tapes. In the 1980s the composer Barry Truax established a method, and consequently also a "style" that dominates the greater majority of compositions with sound grains. According Truax, a large amount of grains is used to create masses of sound (TRUAX, 1988). This method became a common practice to look for high-level physical or computational models to control the synthesis parameters. In line with this approach, in a previous research, for instance, we experimented Genetic Algorithms (SOUZA; MAIA, 2009) as high-level controls to generate sound streams. High-level controls are very useful to create textures in which single grain parameters are not very meaningful but the resulting stream of a combination of many of them according to a high-level parameter model can generate interesting unexpected sounds. On the other hand, Curtis Roads, in his more recent works, articulated words, pulsars and clouds in which he chooses meticulously attack time and the kind of sound grain (ROADS, 2001).

More recently in our creative practice, we used Granular Streamer and Granular Drawer to create an installation in which a diversity of deterministic granular objects was triggered. The algorithm is based on mathematical models as those described in Section 4 and 5 above. These works were presented at "Concertos na Casa do Lago" (Casa do Lago - UNICAMP, Campinas, $10 / 10 / 2011)$, using a cinema screen and stereophonic system for the duration of two hours. The visual objects were shown scrolling upwards representing time evolution (Figure 9).

FIGURE 9 - First experiment in Granular Visual Music using deterministic objects

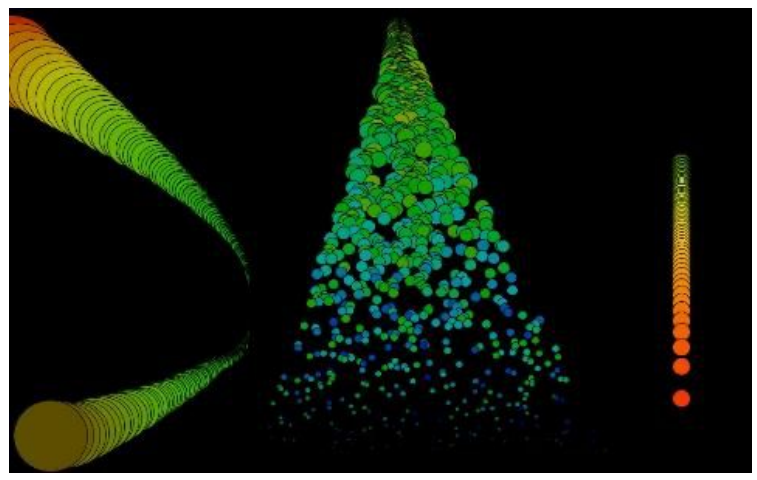

Source: authors 
In our earliest creations, we've also designed textural granular objects using probabilistic distributions and randomness. Using this approach, we created the work Rain which was presented in many events like "I Hear Colours Man! 2" (Greenbank Pub, Bristol, 28/11/2014), "Casa de Pandora 1"(Casa da Luz, São Paulo, 25/07/2014) and Ibr91 (Ibrasotope, São Paulo, 18/09/2015). The concept of Rain is to use audiovisual grains to mimic the textural characteristics of a storm. Those textures are slowly transformed occupying the sound space from silence to white noise. At the visual domain, something similar happens gradually filling a black background until it becomes predominantly white but very turbulent (Figure 10).

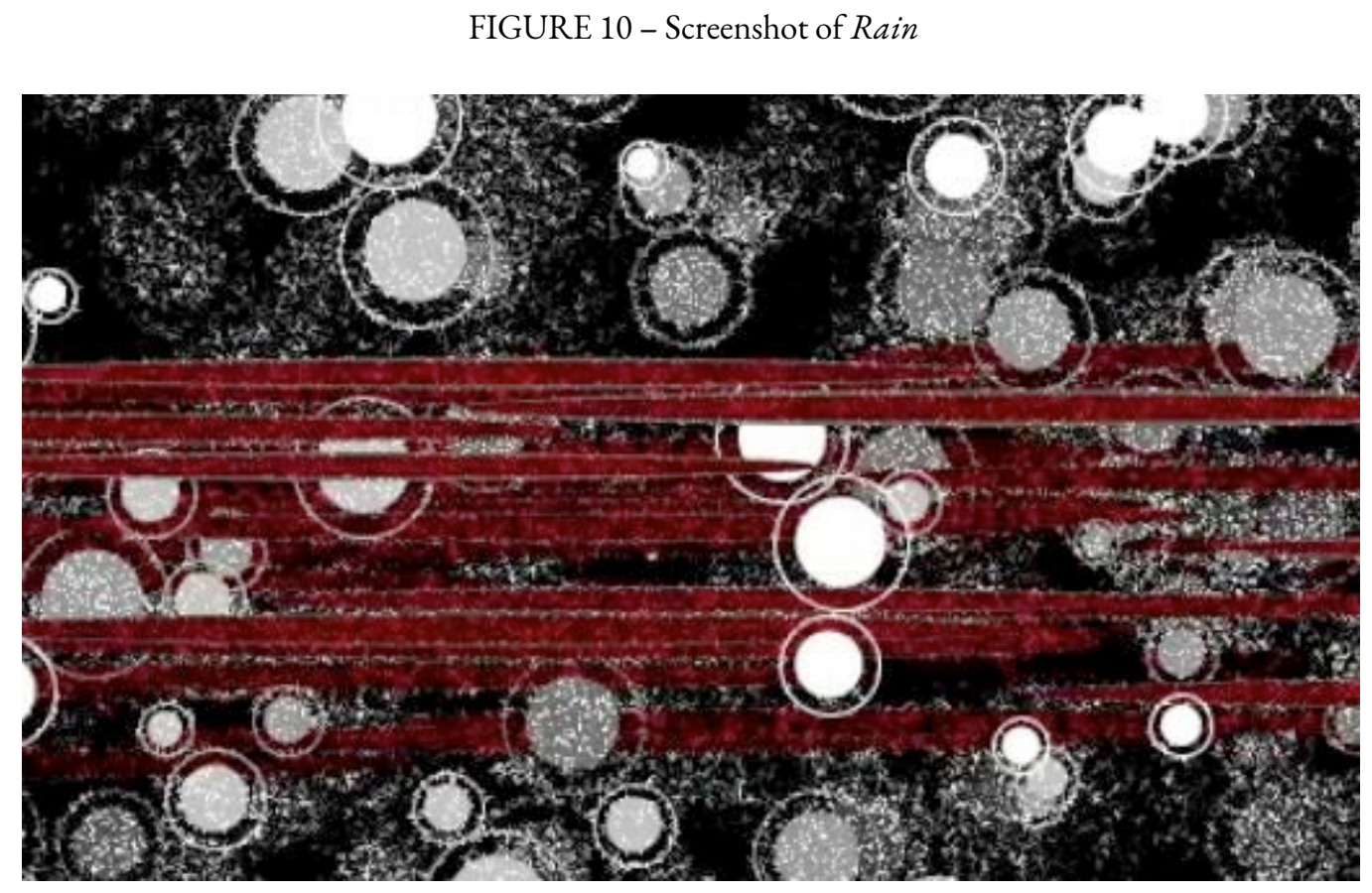

Source: authors

In our third approach to the creative process, we reproduced what Curtis Roads did in the age of early computers, that is, we chose the exact parameter values and time of each grain to compose a set of works named Contrapontos 3, 4, 9 e 8 (Understand Visual Music, 2015). This processed resembled writing traditional music scores, note by note, following a Webern's tendency to use atomistic music material. To do so, we used Granular Score to position each grain at an exact place on the score (Figure 11). It is possible to notice how flexible granular compositions can be, allowing a large diversity of distinct control strategies. 
FIGURE 11 - Granular score of Contrapontos.

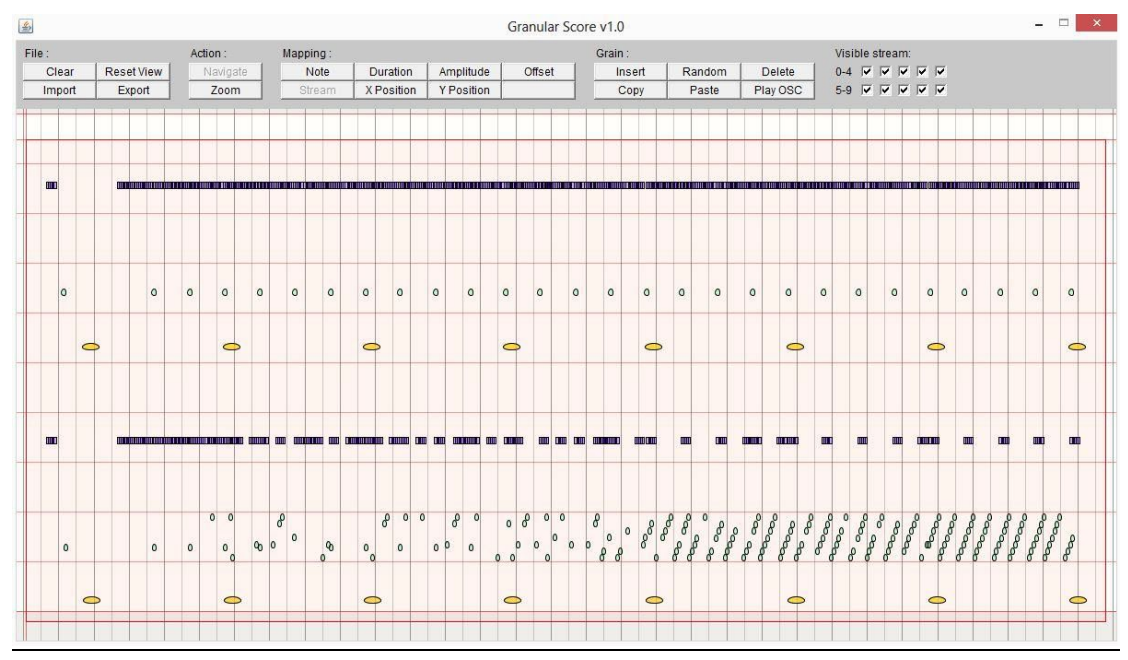

Source: authors

The main goal of our research has always been the creative practice, that is, to create live performances, interactive installations, and fixed media videos. When it comes to create larger works concerning its duration, it becomes harder to attract and hold the public attention. In long music compositions like symphonies and soundtracks, we are used to listen to a diversity of elements in a single work. So, in general, the composer uses different sections of a major piece with a variety of melodic, harmonic, homophonic, polyphonic, and rhythmic characteristics in order to capture the attentiveness of the audience.

The combination of deterministic mathematical models, probabilistic distributions, and grain-by-grain manual control, enabled by the combination of MATLAB, Granular Streamer, Granular Drawer and Granular Score, constitutes the main innovation of our proposal. This multiapproach strategy in one bundled system allowed our creativity to compose long duration performances and installations of Granular Visual Music moving towards the strategies used in traditional music composition.

All the citations of our works on Granular Visual Music can be watched at: https://www.youtube.com/playlist?list=PLYgqh-ao9ZWimtNRG6Cjinv2jfEwmkhhl 


\section{Conclusions and Perspectives}

Since the Xenakis' first mathematical/statistical approach to Granular Synthesis in Music Composition the computer power and software facilities has grew exponentially as well the many formal models for music. For granular synthesis a lot of formal and artistic works has been developed and the trend continues until now. So, we have presented in this work a new integrated system to generate both, deterministically or probabilistically, granular sound structures (streams) with algorithms coded in MATLAB to model grains parameters evolving in time. In other words, sound structures are coded in a simple text file which is interpreted in our software Granular Streamer, generating sound streams. Composition with a set of streams is made through our visual interface Granular Score which sends information to Granular Streamer. We have also developed a MATLAB script for free drawing curves on a window. These curves in Granular Score, considered as time functions of grain's parameters, are "sound gestures" which can be mapped onto granular sound streams. The third part Granular Drawer allows artistic visual representation of generated sound streams.

For the case of statistical models, we've experimented in our research, we found that correlation coefficient of samples of grains, generated through a probability distribution function, has a small impact on the variability of granular sound streams. More important for stream variability are the mean and standard deviation magnitude. However, statistical models must be tested in several regimes and combination of the parameters before more conclusive results can be achieved.

Due the huge number of possibilities to construct formal/mathematical systems as well creative interfaces, there are plenty of room for new explorations as well many experiments in music composition accompanied to visuals (visual music). Our work goes in this direction showing how to construct a simple, but effective audiovisual integrated system based on granular synthesis. 


\section{ACKNOWLEDGMENTS}

The authors thank the Interdisciplinary Nucleus for Sound Communications (UNICAMP) for the hospitality and where most of this research was developed, as well as anonymous referees for the improvement of this work.

\section{REFERENCES}

CIUFO, T., Real-time sound-image manipulations and mapping in a performance setting, In: Digital Arts and Culture Conference. Providence, Rhode Island, 2001.

COLLOPY, F., Playing (with) color, Glimpse: the Art Plus Science of Seeing, Vol. 2.3, p. 62-67, 2009. Also disposable at: http://files.rhythmiclight.com/biblio/collopy2009.pdf.

DI SCIPIO, A., Formalization and Intuition in Analogique A et B, Proceedings of the International Symposium Iannis Xenakis (Athens), Makis Solomos et all (Editor), 2005.

FRAÇOIS, J., Organization of Scattered Timbral Qualities: A Look at Edgard Varèse's Ionisation, Perspectives of New Music, Vol. 29, No. 1 (Winter), p. 48-79, 1991.

GABOR, D. Acoustical quanta and the theory of hearing. Nature 159 (4004), pp. 591-594, 1947.

HILLER, H., ISAACSON, L., Experimental Music, Composition with an Electronic Computer, McGraw-Hill Book Company Inc., 1959.

KAPUCINSKI, J., Basic theory of intermedia: Composing with sounds and images. Monochord: De Musica Acta, Studia e Comentarii 19, p. 43-50, 1998.

MANNING, P., Sound Synthesis Using Computers, in The Oxford Handbook of Computer Music, Dean, R. (Ed.), Oxford University Press, 2011.

MIRANDA, E.R. Granular Synthesis of Sounds by Mean of a Cellular Automaton. Leonardo, Vol. 28, No. 4, p. 297-300, 1995.

RAMAKRISHNAN, C., Illposed Software - JAVA OSC (2016), See internet page: https://github.com/hoijui/JavaOSC Last viewed on Jun, 24, 2021.

ROADS, C. Microsound. MIT Press, 2001.

SOUZA, F. F., MAIA, A.: Evolutive Processes for Granular Synthesis in Extended Gabor Spaces. VII Congresso de Engenharia de Audio (AES), 2009.

SQUIBBS, R., Images of Sound in Xenakis's Mycenae-Alpha, Journées d'Informatique Musicale, île de Tatihou, France. May 1996, hal-02986061. Online Version at: https://hal.archivesouvertes.fr/hal-02986061/document

THIEBAUT, J., HEALE, P., KINNS, N.B., Drawing electroacoustic music. In: Proceedings of ICMC 2008. Ann Arbor, USA, 2001. 
TRUAX, B., Real-Time Granular Synthesis with a Digital Signal Processor, Computer Music Journal 12(2), p. 14-26, 1988.

WEHINGER, R., Artikulation, by G. Ligeti. Graphical Score. See internet page: https://www. youtube.com/watch?v=71hNl_skTZQ. Last view on Jun, 24, 2021. Also see: https://www.semanticscholar.org/paper/SUM-\%3A-DE-LA-SONIFICATION-D\%27IMAGE\%C3\%80-LA-COMPOSITION-Adhitya-

Kuuskankare/15eb384a2b0c7d06d9edd7c538100dd9be07442f/figure/9 . Last view on Jun, 24, 2021.

XENAKIS, I., Formalized Music: Thought and Mathematics in Composition, Harmonologia Series, Pendragon Press, 1992.

\section{ABOUT THE AUTHORS}

Fernando Falci de Souza has a Doctor in Music (2016) by University of Campinas, in the area of Technological Interfaces and Musical Processes. He had research stages at Centre for Musical Research, Bath Spa University (UK) on Visual Music and at Input Devices for Music Interaction Lab (IDMIL), McGill University (Canada) on design and construction of Digital Musical Instruments. He is graduated both in Popular Music by Faculdade Santa Marcelina, and in Computer Science by Universidade de São Paulo. Granular Visual Music is the focus of his research in which he creates audiovisual works of art from correlations of tiny fragments of sound and image. ORCID: https://orcid.org/0000-0002-8605-8531. E-mail: fernando_ffs@yahoo.com.br

Adolfo Maia Jr. is a retired professor from the University of Campinas (UNICAMP). He holds a Dr. Sc. in Theoretical Physics. He was invited researcher at the University of Oxford (Theoretical Physics), Brown University (Astrophysics and Cosmology) and University of Plymouth (Computer Music). He is currently invited researcher at the Interdisciplinary Nucleus for Sound Communications (NICS/UNICAMP) and his research is in the area of Formal/Mathematical Models for Music Composition and Analysis. ORCID: https://orcid.org/0000-0001-7224-8310. E-mail: maia.adolfo@gmail.com 